\title{
Lower albumin levels are associated with frailty measures, trace elements, and an inflammation marker in a cross-sectional study in Tanushimaru
}

\author{
Maki Yamamoto ${ }^{1}$, Hisashi Adachi ${ }^{2 *}$, Mika Enomoto ${ }^{1}$, Ako Fukami ${ }^{1}$, Sachiko Nakamura', Yume Nohara',
} Akiko Sakaue', Nagisa Morikawa', Hitoshi Hamamura' ${ }^{1}$, Kenta Toyomasu ${ }^{1}$ and Yoshihiro Fukumoto ${ }^{1}$

\begin{abstract}
Background: There is little data on the association between the lower nutrition represented by serum albumin levels and related factors in a general population. The present study aimed to determine whether the albumin level positioned as some kind of biomarker with frailty measures, trace elements, and an inflammation marker.

Methods: In 2018, we performed an epidemiological survey in 1368 subjects who resided in Tanushimaru, Japan, in which we examined the blood chemistry including albumin, trace elements, hormone levels, and carotid ultrasonography. Albumin levels were categorized into 4 groups (G1 [3.2-3.9 mg/dL], G2 [4.0-4.3 mg/dL], G3 [4.4$4.6 \mathrm{mg} / \mathrm{dL}]$, and G4 [4.7-5.3 mg/dL]). The participants underwent measurements of handgrip strength and were tested by asking to walk $5 \mathrm{~m}$. Their cognitive functions were evaluated by the mini-mental state examination (MMSE).

Results: Multiple stepwise regression analysis demonstrated that albumin levels were significantly and independently associated with age (inversely), systolic blood pressures, estimated glomerular filtration rate (eGFR), MMSE score, frailty measures (handgrip strength), an inflammation marker (high-sensitivity C-reactive protein), hormones (growth hormone (inversely) and insulin-like growth factor-1), and trace elements (calcium, magnesium, iron, and zinc), with a linear trend.
\end{abstract}

Conclusions: Lower albumin levels, even in the normal range, were found to be related factors of frailty measures, trace elements, and an inflammation marker in a general population.

Keywords: Albumin, Frailty measures, Cognitive function, Trace elements, Epidemiology

\section{Background}

It has been reported that hypoalbuminemia is associated with greater all-cause mortality in a general population $[1,2]$. We have also reported the impact of lower albumin levels on long-term mortality in our cohort [3]. The

\footnotetext{
* Correspondence: hadac@med.kurume-u.ac.jp

${ }^{2}$ Department of Community Medicine, Kurume University School of Medicine, 67 Asahi-machi, Kurume 830-0011, Japan

Full list of author information is available at the end of the article
}

normal serum concentration of albumin in healthy adults is $\geq 4.0 \mathrm{~g} / \mathrm{dL}$, while hypoalbuminemia is defined as a serum albumin level of $\leq 3.4 \mathrm{~g} / \mathrm{L}$ [4]. A metaanalysis by Vincent et al. [5] suggested that hypoalbuminemia is an independent risk factor in patients with acute illness. Hypoalbuminemia was associated with frailty [6], cognitive impairment [7], and trace elements deficiency [8]. It was demonstrated that low serum albumin is a risk factor for frailty in elderly people with

(c) The Author(s). 2021 Open Access This article is licensed under a Creative Commons Attribution 4.0 International License, which permits use, sharing, adaptation, distribution and reproduction in any medium or format, as long as you give appropriate credit to the original author(s) and the source, provide a link to the Creative Commons licence, and indicate if changes were made. The images or other third party material in this article are included in the article's Creative Commons licence, unless indicated otherwise in a credit line to the material. If material is not included in the article's Creative Commons licence and your intended use is not permitted by statutory regulation or exceeds the permitted use, you will need to obtain permission directly from the copyright holder. To view a copy of this licence, visit http://creativecommons.org/licenses/by/4.0/. The Creative Commons Public Domain Dedication waiver (http://creativecommons.org/publicdomain/zero/1.0/) applies to the data made available in this article, unless otherwise stated in a credit line to the data. 
diabetes in a Japanese cross-sectional study [9]. In the longitudinal study, lower levels of serum albumin are associated with future decline in functional performance in older people [10]. However, most reports have been limited to older subjects $[6,7]$ and to older hospital patients [8]. The age of the target cases in previous papers $[6,7]$ was over 60 years old.

Reports with association between hypoalbuminemia and growth hormone $(\mathrm{GH})$ or insulin-like growth factor1 (IGF-1) were also scant. A study of hemodialysis patients revealed that hypoalbuminemia was significantly associated with lower IGF-1 [11]. In the setting of malnutrition, GH was positively, and IGF-1 was negatively, associated with inflammation markers such as highsensitivity C-reactive protein (Hs-CRP) [12], suggesting that inflammatory markers were independent predictors of frailty [13].

The above-mentioned studies were all clinical and dealt with older adults and patients. Therefore, we aimed to determine whether lower albumin levels $(<4.0 \mathrm{mg} /$ $\mathrm{dL}$ ) are associated with frailty measures, trace elements, an inflammation marker, and nutrition intake in a large number of healthy subjects from a Japanese general population in the present study.

\section{Methods}

\section{Subjects}

We enrolled subjects from the typical farming town, Tanushimaru, located in Kyushu, the southwestern island of Japan conducted in 2018 as a Japanese cohort of the Seven Countries Study [14]. The subjects of this study are 1368 participants (554 males and 814 females: mean age of 68.8 years) of a health check-up examination. As previously reported, the demographic background of the subjects in this area is similar to that of the general Japanese population [15].

\section{Data collection}

The subjects' medical history, history of cardiocerebrovascular diseases, use of alcohol, and smoking were ascertained by a questionnaire. Alcohol intake and smoking were classified as current habitual use or not. Height and weight were measured, and body mass index (BMI) was calculated as weight (kilograms) divided by the square of height (square meters) as an index of obesity. Waist circumference was measured at the level of the umbilicus in the standing position. Blood pressure (BP) was measured in the supine position twice at 3-min intervals using an upright standard sphygmomanometer. Vigorous physical activity and smoking were avoided for at least $30 \mathrm{~min}$ before $\mathrm{BP}$ measurement. The second $\mathrm{BP}$ with the fifth-phase diastolic pressure was used for analysis. Hypertensive subjects were defined as those with systolic BP $\geq 140 \mathrm{mmHg}$ and/or those with diastolic BP $\geq 90 \mathrm{mmHg}$ and/or those receiving antihypertensive medication. Subjects with fasting plasma glucose (FPG) $\geq 6.99 \mathrm{mmol} / \mathrm{l}(126 \mathrm{mg} / \mathrm{dl})$ and/or subjects taking oral hypoglycemic agents or receiving insulin injection were diabetic. Subjects with dyslipidemia were defined as those with low-density lipoprotein cholesterol (LDL-c) $\geq$ $3.62 \mathrm{mmol} / \mathrm{l}(140 \mathrm{mg} / \mathrm{dl})$ and/or triglycerides $\geq 1.69$ $\mathrm{mmol} / \mathrm{l}(150 \mathrm{mg} / \mathrm{dl})$ and/or high-density lipoprotein cholesterol (HDL-c) $<1.03 \mathrm{mmol} / \mathrm{l}(40 \mathrm{mg} / \mathrm{dl})$ and/or those taking lipid-lowering drugs.

Fasting blood samples were centrifuged within $1 \mathrm{~h}$ after collection. Serum levels of IGF-1 and GH were measured by ELISA and ECLIA methods to 1363 subjects who could receive blood testing. The blood was submitted to the commercially available laboratory (SRL Inc. Fukuoka, Japan), and the intra- and inter-assay coefficient of variations of IGF-1 and GH, respectively, at the laboratory that performed the assays was $2.56 \%$ and $0.75 \%$, and $3.06 \%$ and $0.73 \%$ [16]. Liver enzymes (alanine aminotransferase [ALT], aspartate aminotransferase [AST], and $\gamma$-glutamyl transpeptidase $[\gamma$-GTP]) were also measured. Estimated glomerular filtration rate (eGFR) was calculated by the following estimation formula that has been recommended by the Japan Society of Nephrology: eGFR $\left(\mathrm{ml} / \mathrm{min} / 1.73^{2}\right)=\left(194 \times \mathrm{Scr}^{-1.094}\right.$ $\left.\times \operatorname{age}^{-0.287}\right) \times(0.739$ for females $)$ [17]. In addition to these blood testing, trace elements (calcium, magnesium, iron, zinc, and phosphorus) and Hs-CRP as an inflammation marker were measured. The participants underwent measurements of handgrip strength using a calibrated strain-gauged dynamometer and expressed in kilograms $(\mathrm{kg})$. They were asked to squeeze maximally with the dominant hand. The score of the dominant hand was used for analysis. Walk speed was measured over a 5-m course, marked out on level ground. Participants were asked to walk the course at their usual walking pace from a standing start. The time taken to complete the course was timed using a digital stopwatch and recorded to the nearest tenth of a second. The fastest time was used to derive walk speed in meters per second. Their cognitive functions were evaluated by the mini-mental state examination (MMSE). MMSE is designed to quickly measure global cognitive functioning, temporal and spatial orientation, attention, immediate and short-term memory, language, praxis, and calculation. Scores ranged from 0 to 30 , with higher scores indicating better cognitive performance.

Carotid intima-media thickness (c-IMT) of the common carotid artery was determined by using duplex ultrasonography (Sonosite "TITAN," ALOKA) with a $10-\mathrm{MHz}$ transducer in the supine position. Longitudinal B-mode images at the diastolic phase of the cardiac cycle were recorded by a single trained technician who was blinded to the subjects' background. We measured the 
only far wall of c-IMT. The images were magnified and measured on the screen and printed with a highresolution line recorder (LSR-100A, Toshiba). We measured c-IMT according to the originally described method published in circulation [18]. Briefly, the c-IMT defined by Pignoli et al. [18] was measured as the distance from the leading edge of the first echogenic line to the leading edge of the second echogenic line. The first line represented the lumen-intimal interface; the collagen-containing upper layer of the tunica adventitia formed the second line. At each longitudinal projection, the site of the greatest thickness, including plaque, was sought along the arterial walls nearest the skin and farthest from the skin from the common carotid artery to the internal carotid artery. Three determinations of cIMT of one artery were conducted at the site of the greatest thickness and at 2 other points, $1 \mathrm{~cm}$ upstream and $1 \mathrm{~cm}$ downstream from this site. The averaged value among the 6 IMTs (3 from the left and 3 from the right) was used as the representative value for each individual.

This study was approved by the Tanushimaru branch of the Japan Medical Association and by the local mayor, as well as by the ethics committee of Kurume University School of Medicine. All the participants gave informed consent. The Research Ethics Committee of the Kurume University School of Medicine (Process numbers 09019/ 2018) approved the study in conformity with the principles embodied in the declaration of Helsinki.

\section{Statistical analysis}

Because of their skewed distribution, natural logarithmic transformations were performed for the values of triglycerides, Hs-CRP, IGF-1, and GH. These values after the analysis using $\log$ (natural)-transformed values were presented in the original scale (Tables 1 and 2). In Tables 1 and 2, these values are presented as the geometric mean and range. Sex $(\operatorname{men}=0$, women $=1)$, smoking habits (non-smoker and former smoker $=0$, current smoker $=$ 1 ), alcohol intake (non-drinker and former drinker $=0$, current drinker $=1$ ), and medications for hypertension, dyslipidemia, and diabetes (no $=0$, yes $=1$ ) were used as dummy variables. The mean serum albumin levels were classified into the following 4 groups: G1 $(3.2-3.9 \mathrm{mg} /$ $\mathrm{dL}), \mathrm{G} 2(4.0-4.3 \mathrm{mg} / \mathrm{dL}), \mathrm{G} 3(4.4-4.6 \mathrm{mg} / \mathrm{dL})$, and $\mathrm{G} 4$ $(4.7-5.3 \mathrm{mg} / \mathrm{dL})$. The mean parameters stratified by the 4 albumin levels groups were compared using an analysis of variance (ANOVA). Age and sex-adjusted means of parameters stratified by the 4 serum albumin groups were compared using an analysis of covariance (ANCOVA). For the categorical parameters, the $\chi^{2}$ test was used to test differences among groups. Univariate regression analysis was performed using a linear regression analysis. Using the significant factors detected by the univariate analysis, multiple stepwise regression analysis was performed. $P$ values of $<0.05$ were considered to indicate statistical significance. All of the statistical analyses were performed using the SAS software program (version 9.4, SAS Institute, Cary, NC, USA).

\section{Results}

Table 1 shows the characteristics of the 1363 subjects stratified by the 4 serum albumin groups. The characteristics of age $(p<0.001$; inversely), systolic $(p=0.025)$, and diastolic $(p<0.001)$ BPs, total cholesterol $(p<$ $0.001)$, HDL-c $(p<0.001)$, LDL-c $(p<0.001)$, ALT $(p<$ $0.001), \gamma$-GTP $(p=0.036)$, eGFR $(p<0.001)$, alcohol intake $(p=0.003)$, current smoking $(p=0.022)$, MMSE score $(p<0.001)$, handgrip strength $(p<0.001)$, times of walk $5 \mathrm{~m}(p<0.001$; inversely), Hs-CRP $(p<0.001$; inversely), $\mathrm{GH}(p<0.001$; inversely), IGF-1 $(p<0.001)$, calcium $(p<0.001)$, magnesium $(p<0.001)$, iron $(p<$ $0.001)$, zinc $(p<0.001)$, phosphorus $(p=0.025)$, and total IMT ( $p<0.001$; inversely) were significantly associated with the albumin quartiles.

Table 2 shows the age and sex-adjusted means of parameters stratified by the 4 serum albumin groups. The characteristics of systolic $(p<0.001)$ and diastolic $(p<$ $0.001)$ BPs, total cholesterol $(p<0.001)$, HDL-c $(p<$ $0.001)$, LDL-c $(p<0.001)$, ALT $(p=0.004)$, medication for hyperlipidemia $(p=0.008)$, MMSE score $(p=0.002)$, handgrip strength $(p<0.001)$, times of walk $5 \mathrm{~m}(p<$ 0.001 ; inversely), Hs-CRP ( $p<0.001$; inversely), GH $(p=$ 0.017; inversely), IGF-1 $(p<0.001)$, calcium $(p<0.001)$, magnesium $(p<0.001)$, iron $(p<0.001)$, and zinc $(p<$ 0.001 ) were significantly associated with the 4 albumin groups.

Table 3 shows the results of univariate analyses for correlates of serum albumin levels. Age $(p<0.001)$, systolic $(p<0.001)$, and diastolic $(p<0.001)$ BPs, total cholesterol $(p<0.001)$, HDL-c $(p<0.001)$, LDL-c $(p<$ $0.001)$, triglycerides $(p=0.011), \operatorname{AST}(p<0.001), \operatorname{ALT}(p$ $<0.001), \gamma$-GTP $(p=0.011)$, eGFR $(p<0.001)$, alcohol intake $(p<0.001)$, current smoking $(p=0.020)$, MMSE score $(p<0.001)$, handgrip strength $(p<0.001)$, times of walk $5 \mathrm{~m}(p<0.001$; inversely), Hs-CRP $(p<0.001$; inversely), GH $(p<0.001$; inversely), IGF-1 $(p<0.001)$, calcium $(p<0.001)$, magnesium $(p<0.001)$, iron $(p<$ $0.001)$, zinc $(p<0.001)$, phosphorus $(p=0.002)$, and total IMT ( $p<0.001$; inversely) were significantly associated with albumin levels.

Using the significant factors detected by univariate analysis in Table 3, we performed the multiple stepwise regression analysis (Table 4). Eventually, the significances of age $(p<0.001)$, calcium $(p<0.001)$, zinc $(p<$ $0.001)$, magnesium $(p<0.001)$, iron $(p<0.001)$, Hs-CRP $(p<0.001$; inversely), systolic BP $(p<0.001), \mathrm{GH}(p<$ 0.001 ; inversely), IGF-1 $(p=0.004)$, eGFR $(p=0.007)$, 
Table 1 Baseline characteristics stratified by serum albumin groups

\begin{tabular}{|c|c|c|c|c|c|}
\hline Variables & G1 (lowest) & G2 & G3 & G4 (highest) & $P$ for trend \\
\hline Number & 31 & 355 & 632 & 345 & \\
\hline Albumin range $(\mathrm{g} / \mathrm{dL})$ & $3.2-3.9$ & $4.0-4.3$ & $4.4-4.6$ & $4.7-5.3$ & \\
\hline Albumin (g/dL) & $3.8 \pm 0.1$ & $4.2 \pm 0.1$ & $4.5 \pm 0.1$ & $4.8 \pm 0.1$ & $<0.001$ \\
\hline Age (years) & $79.8 \pm 8.8$ & $72.7 \pm 11.4$ & $67.8 \pm 10.9$ & $65.4 \pm 10.5$ & $<0.001$ \\
\hline Sex (\%males) & $15(48.4)$ & $148(41.7)$ & $251(39.7)$ & $136(39.4)$ & 0.722 \\
\hline Body mass index $\left(\mathrm{kg} / \mathrm{m}^{2}\right)$ & $22.3 \pm 3.4$ & $23.2 \pm 3.7$ & $23.1 \pm 3.4$ & $22.9 \pm 3.5$ & 0.486 \\
\hline Systolic blood pressure $(\mathrm{mmHg})$ & $138 \pm 24$ & $136 \pm 20$ & $139 \pm 21$ & $141 \pm 21$ & 0.025 \\
\hline Diastolic blood pressure $(\mathrm{mmHg})$ & $71 \pm 11$ & $77 \pm 11$ & $80 \pm 11$ & $83 \pm 12$ & $<0.001$ \\
\hline Total cholesterol (mg/dL) & $170 \pm 31$ & $199 \pm 32$ & $210 \pm 36$ & $221 \pm 36$ & $<0.001$ \\
\hline HDL-cholesterol (mg/dL) & $52 \pm 12$ & $60 \pm 15$ & $65 \pm 16$ & $70 \pm 20$ & $<0.001$ \\
\hline LDL-cholesterol (mg/dL) & $101 \pm 27$ & $119 \pm 28$ & $126 \pm 31$ & $131 \pm 31$ & $<0.001$ \\
\hline Triglycerides $(\mathrm{mg} / \mathrm{dL})^{\mathrm{a}}$ (range) & 95 (43-199) & $105(30-530)$ & $104(27-878)$ & $108(31-996)$ & 0.467 \\
\hline AST (IU/L) & $23.5 \pm 7.5$ & $23.8 \pm 9.4$ & $24.0 \pm 10.5$ & $25.2 \pm 9.0$ & 0.205 \\
\hline ALT (IU/L) & $17.6 \pm 10.2$ & $18.9 \pm 11.6$ & $20.3 \pm 11.5$ & $23.4 \pm 16.4$ & $<0.001$ \\
\hline Y-GTP (IU/L) & $26.6 \pm 30.8$ & $31.6 \pm 46.0$ & $31.4 \pm 31.1$ & $38.0 \pm 38.1$ & 0.036 \\
\hline eGFR (ml/min/1.73m²) & $60.8 \pm 23.8$ & $66.3 \pm 17.8$ & $70.0 \pm 15.7$ & $71.9 \pm 14.5$ & $<0.001$ \\
\hline Uric acid (mg/dL) & $5.2 \pm 1.3$ & $5.3 \pm 1.4$ & $5.3 \pm 1.3$ & $5.4 \pm 1.4$ & 0.415 \\
\hline $\mathrm{HbA}_{1 \mathrm{c}}(\%)(\mathrm{NGSP})$ & $5.8 \pm 0.7$ & $5.8 \pm 0.6$ & $5.7 \pm 0.5$ & $5.8 \pm 0.6$ & 0.248 \\
\hline Alcohol intake (\%yes) & $11(35.5)$ & $142(40.0)$ & $304(48.1)$ & $183(53.0)$ & 0.003 \\
\hline Current smoking (\%yes) & $0(0.0)$ & $36(10.1)$ & $57(9.0)$ & $48(14.0)$ & 0.022 \\
\hline \multicolumn{6}{|l|}{ Medications } \\
\hline Hypertension (\%yes) & $14(45.2)$ & $142(40.0)$ & $230(36.5)$ & $122(35.5)$ & 0.455 \\
\hline Hyperlipidemia (\%yes) & $10(32.3)$ & $76(21.7)$ & $164(26.4)$ & $91(26.8)$ & 0.253 \\
\hline Diabetes (\%yes) & $5(16.1)$ & $34(9.6)$ & $59(9.4)$ & $40(11.6)$ & 0.471 \\
\hline MMSE (score) & $26.6 \pm 4.2$ & $27.8 \pm 2.8$ & $28.5 \pm 2.1$ & $28.8 \pm 1.8$ & $<0.001$ \\
\hline Grip (kg) & $24.2 \pm 8.2$ & $25.7 \pm 8.9$ & $28.5 \pm 9.1$ & $29.3 \pm 9.2$ & $<0.001$ \\
\hline Walk (sec) & $6.1 \pm 1.9$ & $5.6 \pm 1.6$ & $5.1 \pm 1.2$ & $4.9 \pm 1.1$ & $<0.001$ \\
\hline $\mathrm{Hs}-\mathrm{CRP}(\mathrm{mg} / \mathrm{dL})^{\mathrm{a}}$ (range) & $0.129(0.003-5.370)$ & $0.064(0.003-6.530)$ & $0.045(0.002-1.770)$ & $0.039(0.003-3.830)$ & $<0.001$ \\
\hline Growth hormone (mg/dL) ${ }^{a}$ (range) & $1.40(0.12-8.63)$ & $0.87(0.03-18.5)$ & $0.73(0.03-12.7)$ & $0.61(0.03-13.7)$ & $<0.001$ \\
\hline IGF-1 (mg/dL) a (range) & $80.8(20-241)$ & $88.8(31-233)$ & $96.8(43-216)$ & $102.3(40-223)$ & $<0.001$ \\
\hline Calcium (mg/dL) & $9.0 \pm 0.32$ & $9.34 \pm 0.32$ & $9.56 \pm 0.32$ & $9.83 \pm 0.30$ & $<0.001$ \\
\hline Magnesium (mg/dL) & $2.13 \pm 0.21$ & $2.19 \pm 0.16$ & $2.20 \pm 0.16$ & $2.25 \pm 0.17$ & $<0.001$ \\
\hline Iron $(\mu \mathrm{g} / \mathrm{dL})$ & $72.5 \pm 27.2$ & $90.7 \pm 33.4$ & $99.9 \pm 34.3$ & $108.1 \pm 35.2$ & $<0.001$ \\
\hline $\operatorname{Zinc}(\mu \mathrm{g} / \mathrm{dL})$ & $60.8 \pm 12.9$ & $67.1 \pm 10.6$ & $73.8 \pm 10.7$ & $79.4 \pm 12.2$ & $<0.001$ \\
\hline Phosphorus (mg/dL) & $3.37 \pm 0.64$ & $3.49 \pm 0.51$ & $3.51 \pm 0.48$ & $3.58 \pm 0.45$ & 0.025 \\
\hline Total IMT (mm) & $0.82 \pm 0.26$ & $0.76 \pm 0.28$ & $0.71 \pm 0.18$ & $0.69 \pm 0.16$ & $<0.001$ \\
\hline
\end{tabular}

Data are mean \pm SD or range, unless otherwise indicated

Abbreviations: HDL high-density lipoprotein, $L D L$ low-density lipoprotein, AST aspartate aminotransferase, ALT alanine aminotransferase, $\gamma$-GTP gamma glutamyl transferase, eGFR estimated glomerular filtration rate, $H b A_{1 c}$ glycosylated hemoglobin $A_{1 c}, M M S E$ mini-mental state examination, $H s$-CRP high-sensitivity $C$-reactive protein, IGF-1 insulin-like growth factor 1, IMT intima-media thickness

aLog-transforms values were used for the analysis

handgrip strength $(p=0.013)$, and MMSE $(p=0.024)$ were still remained.

\section{Discussion}

The novel finding of the present study was that the lower albumin level, even in the normal range, was strongly associated with as frailty measures, trace elements, and an inflammation marker in a general population, independent of age.

\section{Lower albumin levels and frailty measures}

We found that handgrip strength, times of walk $5 \mathrm{~m}$, $\mathrm{GH}$, and IGF-1 were significantly associated with the 4 albumin groups. Reports from Smit et al. [6] suggested that serum albumin levels were lower in frail US adults. 
Table 2 Age and sex-adjusted means of parameters stratified by serum albumin groups

\begin{tabular}{|c|c|c|c|c|c|}
\hline Variables & G1 (lowest) & G2 & G3 & G4 (highest) & $P$ for trend \\
\hline Number & 31 & 355 & 632 & 345 & \\
\hline Albumin (g/dL) & $3.81 \pm 0.02$ & $4.21 \pm 0.01$ & $4.50 \pm 0.01$ & $4.80 \pm 0.01$ & $<0.001$ \\
\hline Body mass index $\left(\mathrm{kg} / \mathrm{m}^{2}\right)$ & $22.5 \pm 0.6$ & $23.3 \pm 0.2$ & $23.0 \pm 0.1$ & $22.8 \pm 0.2$ & 0.290 \\
\hline Systolic blood pressure $(\mathrm{mmHg})$ & $131.5 \pm 3.6$ & $134.2 \pm 1.0$ & $139.9 \pm 0.8$ & $142.9 \pm 1.1$ & $<0.001$ \\
\hline Diastolic blood pressure $(\mathrm{mmHg})$ & $72.5 \pm 2.0$ & $77.5 \pm 0.6$ & $80.3 \pm 0.4$ & $82.8 \pm 0.61$ & $<0.001$ \\
\hline Total cholesterol (mg/dL) & $176.1 \pm 6.1$ & $200.5 \pm 1.8$ & $209.0 \pm 1.3$ & $219.1 \pm 1.8$ & $<0.001$ \\
\hline HDL-cholesterol (mg/dL) & $59 \pm 16$ & $64 \pm 16$ & $65 \pm 16$ & $69 \pm 16$ & $<0.001$ \\
\hline LDL-cholesterol (mg/dL) & $105.8 \pm 5.4$ & $121.1 \pm 1.6$ & $125.1 \pm 1.2$ & $129.8 \pm 1.6$ & $<0.001$ \\
\hline Triglycerides (mg/dL)* (range) & $96(43-199)$ & $105(30-530)$ & $104(27-878)$ & $108(31-996)$ & 0.512 \\
\hline AST (IU/L) & $23.1 \pm 1.8$ & $23.6 \pm 0.5$ & $24.0 \pm 0.4$ & $25.3 \pm 0.5$ & 0.126 \\
\hline ALT (IU/L) & $19.2 \pm 2.3$ & $19.6 \pm 0.7$ & $20.2 \pm 0.5$ & $22.8 \pm 0.7$ & 0.004 \\
\hline$\gamma$-GTP (IU/L) & $29.3 \pm 6.6$ & $32.8 \pm 1.9$ & $31.2 \pm 1.4$ & $36.8 \pm 2.0$ & 0.131 \\
\hline eGFR $\left(\mathrm{ml} / \mathrm{min} / 1.73 \mathrm{~m}^{2}\right)$ & $67.4 \pm 2.7$ & $68.6 \pm 0.8$ & $69.5 \pm 0.6$ & $69.9 \pm 0.8$ & 0.622 \\
\hline Uric acid (mg/dL) & $5.03 \pm 0.22$ & $5.27 \pm 0.06$ & $5.28 \pm 0.05$ & $5.44 \pm 0.06$ & 0.107 \\
\hline $\mathrm{HbA}_{1 c}(\%)(\mathrm{NGSP})$ & $5.77 \pm 0.10$ & $5.73 \pm 0.03$ & $5.72 \pm 0.02$ & $5.78 \pm 0.03$ & 0.287 \\
\hline Alcohol intake (\%yes) & $11(35.5)$ & $142(40.0)$ & $304(48.1)$ & $183(53.0)$ & 0.218 \\
\hline Current smoking (\%yes) & $0(0.0)$ & $36(10.1)$ & $57(9.0)$ & $48(14.0)$ & 0.081 \\
\hline \multicolumn{6}{|l|}{ Medications } \\
\hline Hypertension (\%yes) & $14(45.2)$ & $142(40.0)$ & $230(36.5)$ & $122(35.5)$ & 0.219 \\
\hline Hyperlipidemia (\%yes) & $10(32.3)$ & $76(21.7)$ & $164(26.4)$ & $91(26.8)$ & 0.008 \\
\hline Diabetes (\%yes) & $5(16.1)$ & $34(9.6)$ & $59(9.4)$ & $40(11.6)$ & 0.226 \\
\hline MMSE (score) & $27.3 \pm 0.4$ & $28.1 \pm 0.1$ & $28.4 \pm 0.1$ & $28.6 \pm 0.1$ & 0.002 \\
\hline Grip (kg) & $26.1 \pm 1.0$ & $26.8 \pm 0.3$ & $28.3 \pm 0.2$ & $28.4 \pm 0.3$ & $<0.001$ \\
\hline Walk (second) & $5.6 \pm 0.2$ & $5.5 \pm 0.1$ & $5.1 \pm 0.1$ & $5.1 \pm 0.1$ & $<0.001$ \\
\hline $\mathrm{Hs}-\mathrm{CRP}(\mathrm{mg} / \mathrm{dL})^{\mathrm{a}}($ range $)$ & $0.116(0.003-5.370)$ & $0.063(0.003-6.530)$ & $0.045(0.002-1.770)$ & $0.040(0.003-3.830)$ & $<0.001$ \\
\hline Growth hormone (mg/dL) ${ }^{a}$ (range) & $1.19(0.12-8.63)$ & $0.81(0.03-18.5)$ & $0.74(0.03-12.7)$ & $0.66(0.03-13.7)$ & 0.017 \\
\hline IGF-1 (mg/dL) ${ }^{\mathrm{a}}$ (range) & $82.4(20-241)$ & $86.1(31-233)$ & $92.0(43-216)$ & $98.4(40-223)$ & $<0.001$ \\
\hline Calcium (mg/dL) & $8.98 \pm 0.06$ & $9.34 \pm 0.01$ & $9.56 \pm 0.01$ & $9.83 \pm 0.02$ & $<0.001$ \\
\hline Magnesium (mg/dL) & $2.12 \pm 0.03$ & $2.18 \pm 0.01$ & $2.20 \pm 0.01$ & $2.26 \pm 0.01$ & $<0.001$ \\
\hline Iron $(\mu \mathrm{g} / \mathrm{dL})$ & $71.2 \pm 6.1$ & $90.4 \pm 1.8$ & $100.0 \pm 1.3$ & $108.3 \pm 1.8$ & $<0.001$ \\
\hline $\operatorname{Zinc}(\mu \mathrm{g} / \mathrm{dL})$ & $61.0 \pm 2.0$ & $67.1 \pm 0.6$ & $73.8 \pm 0.4$ & $79.3 \pm 0.6$ & $<0.001$ \\
\hline Phosphorus (mg/dL) & $3.45 \pm 0.08$ & $3.51 \pm 0.02$ & $3.50 \pm 0.02$ & $3.56 \pm 0.02$ & 0.241 \\
\hline Total IMT (mm) & $0.75 \pm 0.04$ & $0.73 \pm 0.01$ & $0.71 \pm 0.01$ & $0.71 \pm 0.01$ & 0.239 \\
\hline
\end{tabular}

Data are mean \pm SD or range, unless otherwise indicated

Abbreviations: $H D L$ high-density lipoprotein, $L D L$ low-density lipoprotein, AST aspartate aminotransferase, ALT alanine aminotransferase, $\gamma$-GTP gamma glutamyl transferase, eGFR estimated glomerular filtration rate, $H b A_{1 c}$ glycosylated hemoglobin $\mathrm{A}_{1 c}, M M S E$ mini-mental state examination, $H s$ - $C R P$ high-sensitivity $C$-reactive protein, IGF-1 insulin-like growth factor 1, IMT intima-media thickness

a Log-transforms values were used for the analysis

They defined frailty based on the four domains by Fried et al. [19] as follows: (1) slow walking, (2) muscle weakness, (3) exhaustion, and (4) low physical activity. Lower serum albumin may have been shown to be associated with longer times of walk followed by greater loss of muscle mass and may be an early indicator of impending muscle strength decline as suggested by Schalk et al. [20].

There are many causes of frailty. Of these, alterations in hormones represent major factors involved in the pathophysiology of frailty [21]. The clear-cut effects of GH and IGF-1 on serum albumin levels in healthy individuals have not been demonstrated. Especially, although the effects of $\mathrm{GH}$ on bone and muscle in $\mathrm{GH}$-replete individuals have been studied [22], further investigation remained to be required. Our data in the multiple stepwise regression analyses showed that GH is inversely associated with albumin levels, which can be interpreted by the fact that the declining activity of $\mathrm{GH}$ with age in 
Table 3 Univariate analysis for serum albumin

\begin{tabular}{|c|c|c|c|}
\hline Variables & $\beta$ & SE & $p$ \\
\hline Age (years) & -0.007 & 0.001 & $<0.001$ \\
\hline Sex (\%males) & 0.006 & 0.014 & 0.689 \\
\hline Body mass index $\left(\mathrm{kg} / \mathrm{m}^{2}\right)$ & 0.001 & 0.002 & 0.792 \\
\hline Systolic blood pressure (mmHg) & 0.001 & 0.001 & $<0.001$ \\
\hline Diastolic blood pressure (mmHg) & 0.006 & 0.001 & $<0.001$ \\
\hline Total cholesterol (mg/dL) & 0.002 & 0.001 & $<0.001$ \\
\hline HDL-cholesterol (mg/dL) & 0.004 & 0.001 & $<0.001$ \\
\hline LDL-cholesterol (mg/dL) & 0.002 & 0.001 & $<0.001$ \\
\hline Triglycerides $(\mathrm{mg} / \mathrm{dL})^{\mathrm{a}}$ & 0.034 & 0.014 & 0.011 \\
\hline AST (IU/L) & 0.002 & 0.001 & $<0.001$ \\
\hline ALT (IU/L) & 0.003 & 0.001 & $<0.001$ \\
\hline$\gamma$-GTP (IU/L) & 0.0004 & 0.0002 & 0.011 \\
\hline eGFR $\left(\mathrm{ml} / \mathrm{min} / 1.73 \mathrm{~m}^{2}\right)$ & 0.002 & 0.001 & $<0.001$ \\
\hline Uric acid (mg/dL) & 0.009 & 0.005 & 0.107 \\
\hline $\mathrm{HbA}_{1 \mathrm{c}}(\%)(\mathrm{NGSP})$ & -0.003 & 0.013 & 0.829 \\
\hline Alcohol intake (\%yes) & 0.057 & 0.014 & $<0.001$ \\
\hline Current smoking (\%yes) & 0.053 & 0.023 & 0.020 \\
\hline \multicolumn{4}{|l|}{ Medication } \\
\hline Hypertension (\%yes) & -0.019 & 0.015 & 0.202 \\
\hline Hyperlipidemia (\%yes) & 0.020 & 0.016 & 0.212 \\
\hline Diabetes (\%yes) & 0.017 & 0.023 & 0.457 \\
\hline MMSE (score) & 0.021 & 0.003 & $<0.001$ \\
\hline Grip (kg) & 0.005 & 0.001 & $<0.001$ \\
\hline Walk (sec) & -0.043 & 0.005 & $<0.001$ \\
\hline $\mathrm{Hs}-\mathrm{CRP}(\mathrm{mg} / \mathrm{dL})^{\mathrm{a}}$ & -0.041 & 0.006 & $<0.001$ \\
\hline Growth hormone $(\mathrm{mg} / \mathrm{dL})^{\mathrm{a}}$ & -0.032 & 0.006 & $<0.001$ \\
\hline IGF-1 (mg/dL) $)^{a}$ & 0.237 & 0.021 & $<0.001$ \\
\hline Calcium (mg/dL) & 0.401 & 0.015 & $<0.001$ \\
\hline Magnesium (mg/dL) & 0.279 & 0.041 & $<0.001$ \\
\hline Iron $(\mu \mathrm{g} / \mathrm{dL})$ & 0.002 & 0.001 & $<0.001$ \\
\hline Zinc $(\mu \mathrm{g} / \mathrm{dL})$ & 0.009 & 0.001 & $<0.001$ \\
\hline Phosphorus (mg/dL) & 0.046 & 0.014 & 0.002 \\
\hline Total IMT (mm) & -0.172 & 0.033 & $<0.001$ \\
\hline
\end{tabular}

Abbreviations: $H D L$ high-density lipoprotein, $L D L$ low-density lipoprotein, $A S T$ aspartate aminotransferase, ALT alanine aminotransferase, $\gamma$-GTP gamma glutamyl transferase, eGFR estimated glomerular filtration rate, $H b A_{1 c}$ glycosylated hemoglobin $\mathrm{A}_{1 c}$, MMSE mini-mental state examination, $H s-C R P$ high-sensitivity C-reactive protein, IGF-1 insulin-like growth factor 1, IMT intima-media thickness, SE standard error

a Log-transforms values were used for the analysis

part responsible for better nutritional status [23]. In the final analysis, serum IGF-1 is positively associated with albumin levels. As recent epidemiological survey supported our study [24], it was indicated that lower IGF-1 levels were associated with lower handgrip strength and worse physical performance. This may be also because human aging is accompanied by a decrease in serum IGF-1 secretion.

Cognitive dysfunction is one of the important factors of frailty measures. Our study showed that MMSE score
Table 4 Multiple stepwise regression analysis for serum albumin

\begin{tabular}{|c|c|c|c|}
\hline Variables & $\beta$ & SE & $p$ \\
\hline Calcium (mg/dL) & 0.336 & 0.015 & $<0.001$ \\
\hline Zinc $(\mu \mathrm{g} / \mathrm{dL})$ & 0.005 & 0.0004 & $<0.001$ \\
\hline Age & -0.003 & 0.0006 & $<0.001$ \\
\hline Magnesium (mg/dL) & 0.206 & 0.030 & $<0.001$ \\
\hline Systolic blood pressure (mmHg) & 0.001 & 0.0002 & $<0.001$ \\
\hline $\mathrm{Hs}-\mathrm{CRP}(\mathrm{mg} / \mathrm{dL})^{\mathrm{a}}$ & -0.046 & 0.013 & $<0.001$ \\
\hline Growth hormone $(\mathrm{mg} / \mathrm{dL})^{a}$ & -0.008 & 0.004 & $<0.001$ \\
\hline Iron $(\mu \mathrm{g} / \mathrm{dL})$ & 0.001 & 0.003 & $<0.001$ \\
\hline IGF-1 (mg/dL) a & 0.0003 & 0.0002 & 0.004 \\
\hline $\operatorname{eGFR}\left(\mathrm{ml} / \mathrm{min} / 1.73 \mathrm{~m}^{2}\right)$ & 0.001 & 0.0003 & 0.007 \\
\hline Grip (kg) & 0.002 & 0.001 & 0.013 \\
\hline MMSE (score) & 0.005 & 0.002 & 0.024 \\
\hline
\end{tabular}

Abbreviations: $H s-C R P$ high-sensitivity C-reactive protein, IGF-1 insulin-like growth factor 1, eGFR estimated glomerular filtration rate, MMSE mini-mental state examination, SE standard error

$R^{2}=0.53$

${ }^{\mathrm{a} L o g-t r a n s f o r m s ~ v a l u e s ~ w e r e ~ u s e d ~ f o r ~ t h e ~ a n a l y s i s ~}$

was significantly and independently associated with serum albumin. $\mathrm{Ng}$ et al. reported low albumin was an independent risk marker for cognitive decline in community-living 1664 Chinese older adults [25]. Although the data is limited to elderly hip fracture patients, lower serum albumin levels are independently associated with lower MMSE scores [26].

\section{Lower albumin levels and trace elements}

Of trace elements we have measured, calcium, zinc, magnesium, and iron were positively and significantly associated with albumin levels in the final stepwise regression analysis. It is well known that calcium and iron are closely associated with human nutritional status [27]. On the other hand, Peng et al. [8] suggested serum zinc was a significant independent risk factor for hypoalbuminemia and hospital admissions. Ongan et al. [28] also reported that iron, zinc, and magnesium intake of malnourished elderly persons was lower. Recently, Japanese investigators have suggested that zinc intake was associated with mental disorder, whereas calcium, magnesium, and iron intake were not associated with mental disorders [29]. The impact of dietary mineral intake on mental health is still controversial.

\section{Lower albumin levels and an inflammation marker}

The present study revealed that lower albumin levels are strongly associated with an inflammatory marker such as Hs-CRP. Hypoalbuminemia, which can be associated with various diseases, is frequently observed in dialysis patients, as previously reported [30,31]. One suggested that levels of inflammatory biomarkers such as CRP and 
interleukin-6 are increased in hypoalbuminemic compared with normoalbuminemic dialysis patients [30]. The other suggested that hypoalbuminemia and inflammation may play an important role in atherogenesis [31].

Our previous study suggested that lower albumin levels have a strong impact on cerebro-cardiovascular mortality [3]. The enrolled subjects were free from apparent cerebro-cardiovascular disease at baseline, and their serum albumin levels were within the normal range; however, lower albumin levels and Hs-CRP can affect the high mortality on future cerebrocardiovascular events in this population, because the inflammation is one of the important risk factors for atherosclerosis [32]. The precise mechanism underlying the relationship between lower albumin and an inflammation marker should be clarified in future studies.

Clinical roles of albumin are frequently discussed in hospitalized patients [33-35]. However, there are few reports on the association between lower albumin levels and related factors in a general population. In the clinical practice, lower albumin levels [36] are commonly discovered in association with nutritional deterioration and disease-related inflammatory response. Along with the evolution of the disease itself, this condition might be a result of the aging process, with levels of albumin decreasing with advancing age. However, the association between the lower albumin levels and age has not been fully elucidated; therefore, the association should also take into account diseases and other age-related conditions rather than age alone.

\section{Limitations}

The limitations of this study were as follows. First, because our study design is cross-sectional, we cannot clarify the changes of time trend in albumin. Prospective studies are planned to investigate the role of the factors in the subjects with lower albumin levels in our future studies after long-term follow-up. Second, we performed only a single blood testing to evaluate an association between lower albumin and related parameters. The quadrant shift may occur in the same subjects. Third, the pathophysiological mechanism underlying the association between low albumin levels and related factors was not revealed from our observational study. Nevertheless, clear positive or inverse relationships between lower albumin levels and related factors were striking and further investigation should be required. Fourth, we have no data regarding gastrointestinal tract function in this study.

\section{Conclusions}

Lower albumin levels were found to be related factors of frailty measures, trace elements, and an inflammation marker in a general population.

\section{Abbreviations}

MMSE: Mini-mental state examination; eGFR: Estimated glomerular filtration rate; IMT: Intima-media thickness; GH: Growth hormone; IGF-1: Insulin-like growth factor-1; Hs-CRP: High-sensitivity C-reactive protein; BMI: Body mass index; BP: Blood pressure; FPG: Fasting plasma glucose; LDL-c: Low-density lipoprotein cholesterol; HDL-c: High-density lipoprotein cholesterol

\section{Acknowledgments}

We are grateful to the members of the Japan Medical Association of Ukiha, the elected officials and residents of Tanushimaru, and the team of physicians who helped in performing the health examinations.

\section{Authors' contributors}

Study concept and design: Dr. M.Y.; preparation of the manuscript: Dr. H.A. Acquisition of subjects and/or data: Drs. M.E. and A.F. Performed the examination: Drs. S.N., Y. N., and A.S. Analysis and interpretation of the data: Drs. N.M., H.H., and K.T. Designed the study: Dr. Y.F. The authors read and approved the final manuscript.

\section{Funding}

This study was supported in part by the Kimura Memorial Heart Foundation, Fukuoka, and by Grant-in-Aid for Scientific Research (C) from the Ministry of Education, Culture, Sports, Science and Technology (Grant No. 19K10675).

\section{Ethics approval and consent to participate}

The mayor and the welfare section of Uku town and the Ethical Committee of Kurume University (No. 2284) approved this study. The study conformed to the principles of the declaration of Helsinki. All participants gave informed consent.

Consent for publication

Not applicable, as the study does not contain personal information.

\section{Competing interests}

The authors declare no competing interests.

\section{Author details}

${ }^{1}$ Division of Cardio-Vascular Medicine, Kurume University School of Medicine, Kurume, Japan. ${ }^{2}$ Department of Community Medicine, Kurume University School of Medicine, 67 Asahi-machi, Kurume 830-0011, Japan.

Received: 12 November 2020 Accepted: 4 February 2021

Published online: 19 February 2021

\section{References}

1. Okamura T, Hayakawa T, Hozawa A, Kadowaki T, Murakami Y, Kita Y, Abbott RD, Okayama A, Ueshima H, for the NIPPON DATA 80 Research Group. Lower levels of serum albumin and total cholesterol associated with decline in activities of daily living and excess mortality in a 12-year cohort study of elderly Japanese. J Am Geriatr Soc. 2008;56(3):529-35.

2. Takata Y, Ansai T, Yoshihara A, Miyazaki H. Serum albumin levels and 10-year mortality in a community-dwelling 70-year-old population. Arch Gerontol Geriatr. 2012;54(1):39-43.

3. Umeki $Y$, Adachi $H$, Enomoto $M$, Fukami A, Nakamura $S$, Nohara $Y$, Nakao E, Sakaue A, Tsuru T, Morikawa N, Fukumoto Y. Serum albumin and cerebrocardiovascular mortality during a 15-year Study in a community-based cohort in Tanushimaru, a cohort of the Seven Countries Study. Intern Med. 2016:55(20):2917-25.

4. Herrmann FR, Safran C, Levkoff SE, Minaker KL. Serum albumin level on admission as a predictor of death, length of stay, and readmission. Arch Intern Med. 1992;152(1):125-30.

5. Vincent JL, Dubois MJ, Navickis RJ, Wilkes MM. Hypoalbuminemia in acute illness: is there a rationale for intervention? A meta-analysis of cohort studies and controlled trials. Ann Surg. 2003;237(3):319-34.

6. Smit E, Winters-Stone KM, Loprinzi PD, Tang AM, Crespo CJ. Lower nutritional status and higher food insufficiency in frail older US adults. Br J Nutr. 2013;110(1):172-8.

7. Canon ME, Crimmins EM. Sex differences in the association between muscle quality, inflammatory markers, and cognitive decline. J Nutr Health Aging. 2011;15(8):695-8. 
8. Peng LN, Liang CK, Chou MY, Lin MH, Lai HY, Hwang SJ, Chen LK. Association between serum copper, zinc and hospital admissions among care home residents. Arch Gerontol Geriatr. 2010;51(1):e24-7.

9. Yanagita I, Fujihara $Y$, Iwaya C, Kitajima Y, Tajima M, Honda M, Teruya Y, Asakawa H, Ito T, Eda T, Yamaguchi N, Kayashima Y, Yoshimoto M, Harada M, Yoshimoto S, Aida E, Yanase T, Nawata H, Muta K. Low serum albumin, aspartate aminotransferase, and body mass are risk factors for frailty in elderly people with diabetes-a cross-sectional study. BMC Geriatr. 2020;20: 200.

10. Schalk BW, Visser M, Deeg DJ, Bouter LM. Lower levels of serum albumin and total cholesterol and future decline in functional performance in older persons: the Longitudinal Aging Study Amsterdam. Age Ageing. 2004;33: 266-72.

11. Nilsson E, Carrero JJ, Heimbürger O, Hellberg O, Lindholm B, Stenvinkel P. A cohort study of insulin-like growth factor 1 and mortality in haemodialysis patients. Clin Kidney J. 2016;9(1):148-52.

12. DeBoer MD, Scharf RJ, Leite AM, Férrer A, Havt A, Pinkerton R, Lima AA, Guerrant RL. Systemic inflammation, growth factors, and linear growth in the setting of infection and malnutrition. Nutrition. 2017:33(1):248-53.

13. Harneshaug M, Kirkhus L, Benth JŠ, Grønberg BH, Rostoft S, Bergh S, Hjermstad MJ, Selbæk G, Wyller TB, Kirkevold Ø, Borza T, Saltvedt I, Jordhøy MS. Screening for frailty among older patients with cancer using blood biomarkers of inflammation. J Geriatr Oncol. 2019:10(6):272-8.

14. Kromhout D, Menotti A, Alberti-Fidanza A, Puddu PE, Hollman P, Kafatos A, Tolonen $\mathrm{H}$, Adachi $\mathrm{H}$, Jacobs DR Jr. Comparative ecologic relationships of saturated fat, sucrose, food groups, and a Mediterranean food pattern score to 50-year coronary heart disease mortality rates among 16 cohorts of the Seven Countries Study. Eur J Clin Nutr. 2018;72(8):1103-10.

15. Hirai Y, Geleijnse JM, Adachi H, Imaizumi T, Kromhout D. Systolic blood pressure predicts cardiovascular mortality in a farming but not in a fishing community: a 40-year follow-up of the Japanese cohorts of the Seven Countries Study. Circ J. 2011;75(8):1890-6.

16. Guha N. Assays for GH, IGF-I, and IGF binding protein-3. Methods Mol Biol. 2013;1065:117-28.

17. Matsuo S, Imai E, Horio M, Yasuda Y, Tomita K, Nitta K, Yamagata K, Tomino Y, Yokoyama H, Hishida A. and collaborators developing the Japanese equation for estimated GFR Revised equations for estimated GFR from serum creatinine in Japan. Am J Kidney Dis. 2009;53(6):982-92.

18. Pignoli P, Tremoli E, Poli A, Oreste P, Paoletti R. Intimal plus medial thickness of the arterial wall: a direct measurement with ultrasound imaging. Circulation. 1986;74(6):1399-406.

19. Fried LP, Tangen CM, Walston J, Newman AB, Hirsch C, Gottdiener J, Seeman T, Tracy R, Kop WJ, Burke G, McBurnie MA, Cardiovascular Health Study Collaborative Research Group. Frailty in older adults: evidence for a phenotype. J Gerontol A Biol Sci Med Sci. 2001;56(3):M146-56.

20. Schalk BW, Deeg DJ, Penninx BW, Bouter LM, Visser M. Serum albumin and muscle strength: a longitudinal study in older men and women. J Am Geriatr Soc. 2005;53(8):1331-8.

21. Morley JE, Kim MJ, Haren MT. Frailty and hormones. Rev Endocr Metab Disord. 2005;6(2):101-8.

22. Lissett CA, Shalet SM. Effects of growth hormone on bone and muscle. Growth Horm IGF Res. 2000;10(4):S95-101.

23. Hodes RJ. Frailty and disability: can growth hormone or other trophic agents make a difference? J Am Geriatr Soc. 1994;42(11):1208-11.

24. van Nieuwpoort IC, Vlot MC, Schaap LA, Lips P, Drent ML. The relationship between serum IGF-1, handgrip strength, physical performance and falls in elderly men and women. Eur J Endocrinol. 2018;179(2):73-84.

25. Ng TP, Niti M, Feng L, Kua EH, Yap KB. Albumin, apolipoprotein E-epsilon4 and cognitive decline in community-dwelling Chinese older adults. J Am Geriatr Soc. 2009;57(1):101-6

26. Mizrahi EH, Blumstein T, Arad M, Adunsky A. Serum albumin levels predict cognitive impairment in elderly hip fracture patients. Am J Alzheimers Dis Other Demen. 2008;23(1):85-90

27. White PJ, Broadley MR. Biofortification of crops with seven mineral elements often lacking in human diets--iron, zinc, copper, calcium, magnesium, selenium and iodine. New Phytol. 2009;182(1):49-84.

28. Ongan D, Rakıcıoğlu N. Nutritional status and dietary intake of institutionalized elderly in Turkey: a cross-sectional, multi-center, country representative study. Arch Gerontol Geriatr. 2015;61(2):271-6.

29. Nakamura M, Miura A, Nagahata T, Shibata Y, Okada E, Ojima T. Association between dietary intake and autistic traits in Japanese working adults: findings from the Eating Habit and Well-Being Study. Nutrients. 2019;11(12): 847.

30. Danielski M, Ikizler TA, McMonagle E, Kane JC, Pupim L, Morrow J, Himmelfarb J. Linkage of hypoalbuminemia, inflammation, and oxidative stress in patients receiving maintenance hemodialysis therapy. Am J Kidney Dis. 2003;42(2):286-94

31. Fujii H, Fukuda A, Tanaka M, Kojima Y, Ishida T, Hirata K, Fukagawa M. Putative role of endothelial lipase in dialysis patients with hypoalbuminemia and inflammation. Putative role of endothelial lipase in dialysis patients with hypoalbuminemia and inflammation. Am J Nephrol. 2008;28(6):974-81.

32. Nieto FJ. Infections and atherosclerosis: new clues from an old hypothesis? Am J Epidemiol. 1998;148(10):937-48.

33. Moramarco S, Morciano L, Morucci L, Messinese M, Gualtieri P, Carestia M, Ciccacci F, Orlando S, Buonomo E, Legramante JM, De Lorenzo A, Palombi L. Epidemiology of hypoalbuminemia in hospitalized patients: a clinical matter or an emerging public health problem? Nutrients. 2020;12(12):3656.

34. Akirov A, Masri-Iraqi H, Atamna A, Shimon I. Low albumin levels are associated with mortality risk in hospitalized patients. Am J Med. 2017; 130(12):1465 e11-9.

35. Garwe T, Albrecht RM, Stoner JA, Mitchell S, Motghare P. Hypoalbuminemia at admission is associated with increased incidence of in hospital complications in geriatric trauma patients. Am J Surg. 2016;212(1):109-15.

36. Soeters PB, Wolfe RR, Shenkin A. Hypoalbuminemia: pathogenesis and clinical significance. JPEN J Parenter Enteral Nutr. 2019:43(2):181-93.

\section{Publisher's Note}

Springer Nature remains neutral with regard to jurisdictional claims in published maps and institutional affiliations.

Ready to submit your research? Choose BMC and benefit from:

- fast, convenient online submission

- thorough peer review by experienced researchers in your field

- rapid publication on acceptance

- support for research data, including large and complex data types

- gold Open Access which fosters wider collaboration and increased citations

- maximum visibility for your research: over $100 \mathrm{M}$ website views per year

At $\mathrm{BMC}$, research is always in progress.

Learn more biomedcentral.com/submissions 